\title{
Pulmonary and Extrapulmonary PARAGONIMIASIS in 311 cases Studies in Korea
}

\section{(Preliminary report)}

by

\author{
Graumann Theresia \\ Chief radiologist \\ Swedish - Hospital \\ $1954-1956$
}

PUSAN-KOREA

\author{
Graumann Hans-W \\ Cl: $\cdots$ \\ verman R.C. Hosp. \\ $1951-1956$ \\ Chief radiologist \\ Swedish - Hospital \\ $1956-1957$ \\ PUSAN-KOREA
}

\author{
Shin Sung Woo \\ Assistant radiologist \\ German R. C. Hosp. \\ $1954-1956$ \\ Chief radiologist \\ Medical College of \\ Pusan University \\ PUSAN-KOREA
}

This is a preliminary report as a summary of some lectures on the

"pulmonary and extrapulmonary paragonimiasis."

given at different places itemized below (end of report).

The studies have been made during a period of more than 3 years but are still in progress. We do hope this report may stimulate the curiosity on this subject and may induce further research.

The disease is extremely prevalent in many countries of the orient such as Korea, Japan, Formosa, China, Philippines but has been reported also from Africa, America etc.

On the whole it is striking that medical literature in the past did not describe paragonimiasis as a really serious disease. However, after having seen more than 300 cases, we have reached entirely different conclusions. To date we feel sure that paragonimiasis produces extrenely severe conditions in a great number of patients; and we are convinced that an unexpectedly large number of patients die from the disease with the true diagnosis unrecognized.

This conception is aided by our animal experiments, too.

The interpretation of the many unusual X-rays on Korean patients led us to an extensive research on paragonimiasis, including animal experiments as well as studies on parasitology and epidemiology.

\section{Parasitology:}

Paragonimiasis is a helminthic infection caused by the trematode "Paragonimus Westermani". Westerman, the Director of the Zoological Garden in Amsterdam, found the first parasite in the lungs of a Bengal tiger in 1877 , reported by Kerbert in 1878.

Since that time more than 400 reports 
in paragonimiasis have been published, Because the lungs of mammals most frequently are infected with this fluke, paragonimiasis is also referred to as 'lung-flake-disease", "pulmonary disto miasis" and "endemic hemoptysis", the latter because of the observation that many time patients have rust-coloured, bloody sputum.

The life-cycle of this trematode has been described in many excellent papers by parasitologist. Two intermediate hosts are necessary: first intermediate hosts are snails; second intermediate hosts are crabs and crayfish, bearing the infective agent so "called "metacercaria" which is an early stage of the parasite.

After having eaten improperly cooked or only salted meat of such crabs or crayfish, preparations which do not kill the metacercaria, one gets infected.

Within 8 weeks the migration and maturation of the fluke is considered to be completed. Understandable to us was the fact that many patients denied having eaten crabs or crayfish at all. This problem led us finally to some experiments on metacercariae, isolated from the crab. The resistance of metacercariae, exposed to chlorine 5 p.p.m., has been exciting and showed a survival-rate of at least 11 days. These experiments have been made with the assistance of $\mathrm{Cpt}$. Sanchez, U. S. Army, Area-Surgeon, Pusan.

Such a striking resitance becomes still more important since Yokogawa showed living metacercariae in the scratches of wooden cutting-boards on which crabs previously have been prepared. Therefore the highly important conclusion has to be taken into consideration that infection with paragonimiasis is possible without having eaten the crabs, because remaining metacercariae can contaminate any food and that even after some days due to their resistance-prepared only on the same cutting-boards previously used for the crabs.

A prevention is only efficacious by boiling the crabs and the crayfish sufficiently; and that has to be done before cutting them to prevent a spread of living metacerariae.

After our studies it is advisable to boil any kind of crabs even salt-water-crabs in paragonimus countries. Until now only fresh-water-crabs and crayfish are described as infectious second intermediate hosts. We are the first to find metacercariae in salt-water-crabs. The species was "macrophthalmus de Haan" in which we found them repeatedly. Animal experiments with these "salt-water-me tacercariae" caused the spontaneous death of two young dogs (puppies), infected per mouth. Death took place 5 weeks after the infection. They developed the same radiologic-clinical pictures as observed by us in dogs infected with "fresh-watermetacercariae".

These" salt-water-metacercariae" look quite similar to those of fresh-water-crabs and we bel ieved at first they were immature meta cercariae of paragonimus westermani. However, M. Yokogawa who kindly undeftook a critical examination on these salt-water-metacercariae believes that they represent: a new species. Further experiments started by us shall discover these differences between the radiologic-clinical findings-simulating a typical paragonimiasis in dogs and the contradicting parasitological conception.

Parasitologically it might be also inte- 
resting that othar species of salt-watercrabs collected several times at the same place as the above mentioned "macropthalmus de Haan" did not have metacercariae.

\section{Epidemiology:}

A medical survey of an endemic area at Hadong in the province "Kyong Sang Namdo", not yet marked in previously published maps of the distribution of paragonimiasis in Korea, has been made with assistance of the Swedish Hospital. (Yusan) in oxiober 1955, We examined 400 patients and surprisingly found 70 patients with ova in the sputum in only one examination. The infection-rate is probably much higher with repeated examinations.

The sex-distribution is of special interest; in all our patients in the endemic area as well as in the patients investigated in the Hospital we observed a preponderance of paragonimiasis in males with $80 \%$ while females were infected in only $20 \%$. Eating and drinking habits of males which are implicated some times in clonorchiasis can not be blamed for this sexdistribution particularly since the same percentage exists among children, It seems aiso questionable to us if the practice of catching the crabs by boys causes this sex-distribution since the girls are preparing the crabs and have a sweet tooth.

We made a brief survey of the single case-reports in literature and found there also the inexplicable sex-distribution.

The high infection-rate in children up to 15 years of age witi $22 \%$ of our cases is another important cpideminlngical fart. Since collection of sputum is difficult in this age group the percentage most probably is still higher. Therefore many cases in children could not be confirmed in spite of a high suspicion of paragonimiasis in the chest $\mathrm{X}$-rays.

Moreover the high infection-rate is of importance because we discovered that this disease produces very often in children especially the severe condition and with special $\mathrm{X}$-ray findings described below. This conception is aided also by ou vbservation in animals: young animals did not survive in our experiments. Surprisingly literature did not report these related facts in children and young animals. Only personal information obtained from the 406th Med. Gen. Laboratory of the U.S. Army in Tokyo where Ritchie and Lim developed a skin test of paragonimiasis confirmed our observation. It was told to us that "young animals did not stand the infection" and that therefore older animals had been used for experimental studies.

The observation in both the serious radiologic-clinical condition in children and the fatal outcome in young animals gives at least authority to our claim that paragonimiasis is a most dangerous infection in childhood and causes death many times probably with the true diagnosis unrecognized. This conception is not only important but contradicts especially the previously held beliefs that paragonimiasis is supposed to be relatively benign and that death is rerely attributable to it,

And there are a good many finding that the same conclusin is true for adults, too,

\section{Radiologic-clinical findings:}

The confirmation of paragonimiasis 
depends until now on the discovery of the parasite or its ova. All our patients have been proved in this respect. But we observed that the patients many times do not produce the sputum or ova in it, but only occasionally demonstrate ova. The initial diagnosis with the radiological methods, therefore acquires an important significance. We have had patients in whom this initial diagnosis of paragoni- miasis made sputum investigations advisable but the ova could be demonstrated some times only after repeated search for weeks and months.

Careful investigations led us to the many possible complications caused by pargonimiasis. These complications in pulmonary and extrapulmonary paragonimiasis of our cases are collected in the different groups.

Table 1.

PARAGONIMIASIS

Complications

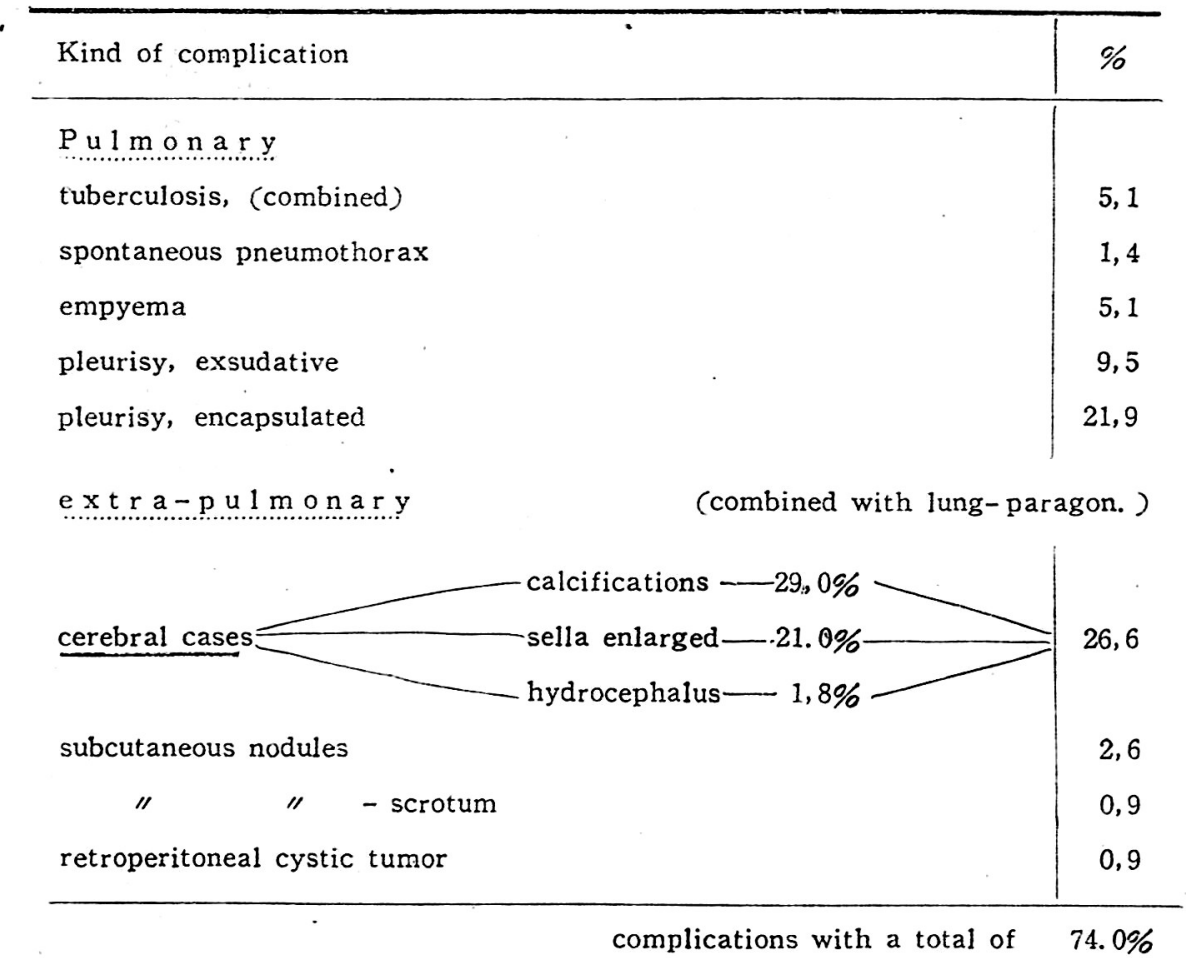

As seen in this table 1 , surprisingly our patients showed only $5 \%$ complicating active tuberculosis, contradicting existing beliefs, But spontaneous pneumothorax, non-specific empyema and exudative or encapsulated pleurisies were significant with a total of $33 \%$.

As a complete surprise more than $25 \%$ of our cases had cerebral complications. Subcutaneous nodules and retroperitoneal or abdominal tumors were rare complications in our material.

\section{Pulmonary Paragonimiasis- classification:}

For the classification of the pulmonary 
paragonimiasis we decided on a certain radiologic-clinical scheme, as shown in

table 2.

PARAGONIMIASIS.

Radiologica! systematics - scheme.

The following findings are usually combined:

Preural reaction, small ${ }_{\text {both sides }}^{\text {one side }}$

$\left.\begin{array}{l}33 \% \\ 34 \%\end{array}\right\} 64 \%$

Pleurisy $=$ - encapsulated
2. exsudative

$\left.\begin{array}{r}19.3 \% \\ 8.7 \%\end{array}\right\}$

$28 \%$

Lung collaps

3. spontan. pneumothor.

$\left.\begin{array}{l}1.2 \% \\ 5.8 \%\end{array}\right\} 7 \%$

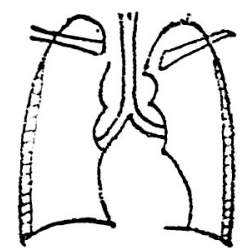

4. empyema

Central process
hilus-mediast. ${ }_{6 .}^{5 .}$ cloudy densities..........

$45.6 \%$

$8.7 \%)$

$45.6 \%$

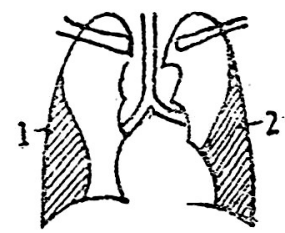

Periphere proc typical

7. mod. apex cyst(Miyake)2.3\%)

cysts, nodules. 8 usual typo

$70.7 \%\}$

$73 \%$

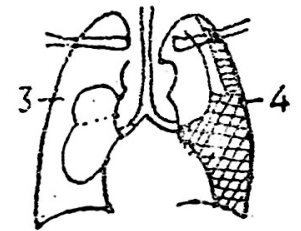

structurc-
-changes

9. bronchopneumonic type

10. bronchiectat. net-type

$\left.\begin{array}{l}16.9 \% \\ 34.1 \%\end{array}\right\}$

$51 \%$
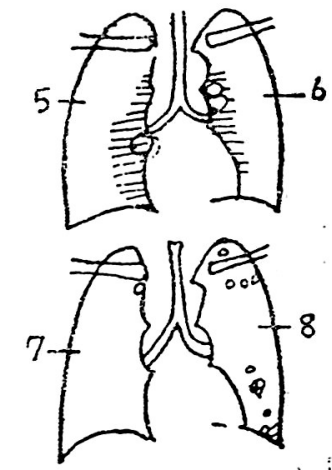

This scheme has demonstrated in seve-

except for a little higher number of a lectures since April 1956.

in the meantime-during about 1 yearthe number of patients has increased but the percentage did not change very mich spontaneous pnemothoraxes and hidden "Miyake-cysts",

This sheme demonstrates 6 maingroups and 12 subgroups, The percentage in 
each group and subgroup is related to the total number of patients. Many $t$ imes the groups have been combind in the same patient.

Our attention is first fccused on smallest lateral pleural shadows which we found in $61 \%$, with the bilateral involvement predominating, comparable pleural are rare in tuberculosis from our experience.

But even more striking unilateral or bilateral changes, like pleurisies-28\% in our cases-suggest paragonimiasis if the patient comes from a "paragonimus-country,". Encapsulated (1) and exudative (2) pleurisies therefore make careful examinations also for paragonimiasis advisable. We saw patients with such pleurisies comparable to a tuberculosis, but the diagnosis of a paragonimiasis, initially in $\mathrm{X}$ rays, could be proved by ova in the sputum, the stool and/or the pleural fluid while all examinations for tuberculosis-including cultures-had been negative. Such pleurisies can develop also in the mediastinum and the lung-base.

Spontaneous pneumothorax (3), slowly or sudden developing, could be observed by us in men as well as in animals. We do not agree with Miyake who maintained spontaneous pneumothorax to be an early symptom of paragonimiasis in dogs; on the contrary, we observed it always as an additional affliction, which can appear consecutively even after a longstanding typical paragonimiasis.

Through the studies of Kjaergaard and others we know that tuberculosis is, percentagewise, a rather uncommon cause of spontaneous pneumothorax, but that the so called "idiopathic pneumothorax" is most common in many countries.
Additionally we have to state that a real "symptomatic paragonimus-pneumothorax" has to be taken into consideration in "paragonimus countries",

Empyemas (4) with 6\%ó in our cases are also of particular interest and may often arise from the above mentioned pleurisies or pneumothoraces.

The percentage of this conditions is expected to be much higher; but certain circumstances and the overwhelmingly high number of patients in the hospitals made it impossible to us to follow up all those empyemas suggestive of paragonimiasis. Our 6\% are proved by ova in the sputum and/or the empyema fluid, of course tuberculosis has been excluded.

Death probably takes place in a high number of those empyemas, may be with the true diagnosis unrecognized, especially in outlying districts,

Most important are the next groups (5/6) with a central-mediastinal-hilar affliction in about $45 \%$ of our cases. Literature surprisingly never describes such central-form as typical for paragonimiasis. We found the very impressive changes in all age groups but extremely preponde-rantly in children.

In animal experiments we saw the same mediastinal changes in a puppy.

Clinically the patients show a tendency to a more serious condition.

The fatal outcome is not unusual as we could prove in children. They often die unexpectedly fast. Those cases had had been particularly misinterpreted as tuberculosis, and got the corresponding treatment but finally were proved to be a paragonimiasis.

The X-rays of such children also demonstrate a malisnant picture with large and dense hilar regions of a nodular 
confluent type, simulating a central pneumonia or a mediastinal inflammation etc, , some times with an enlarged heart. The diagnosis has been very difficult in all radiological points of view, since the central form has not been described yet in literature as pathognomonic for paragonimiasis and since the patient many times does not demonstrate ova in the sputum and/or the stool.

In this regard the 406 th $\mathrm{Med}$. Gen. Laboratory of the U. S. Army, Tokyo, -Chief pathologist Col. Blumberg-gives an important viewpoint in a microscopic section of the lungs in our animal experiments: namely ova were seen-in one dog-only "within the lining and in the surrounding tissue of the cysts". Even if we do not understand why the ova have not been within the cysts, these findings make it understandable that patients can have negative sputa.

The next groups (7/8) with cysts and nodules are described in literature as typical findings on paragonimiasis in chest $\mathrm{X}$-rays. We observed them in $73 \%$ but mostly combined with the other groups. While it seems that patients demonstrate either the cystic or the nodular form we observed that both groups can be present in the same patient. Cysts and nódules are often close together and confluent, but cysts can become nodular forms and vice versa. They are often relatively well outlined but are to be found some times within hazy or patchy densities,

Pathognomonic for paragonimiasis is the tendency for such lesions to stay unchanged for a long time.

Out of this group it seems important to emphasize the so called, hidden, "Miyakecyst" (7) with a paramediastinal localis- ation in the upper lung-field. We proposed this name honoring the excellent description by Miyake in 1951. This cyst mostly can be outlined only in a bodysection-radiography ("tomography").

Many of the so called X-ray-negative cases in literature probably are such hidden "Miyake-cysts".

In structure-changes we find next the rbonchopneumonic type (9). The scattered groups of mottled, densifies suggestive of bronchopneumonia, are very typical. With a more hazy appearence, they look less malignant than a real bronchopneumonia, The densities are more scattered. and are often combined with one of the other groups of the scheme.

The bronchiectatic form (10) with a webby structure makes it understandable that literature should confuse such pictures with the true bronchiectasis. But clinically, as well as radiologically and pathologically this seems to be a mistake. Due to considerations for principle, we did not extremely study this question since bronchography or chest surgery seems to us mostly contraindicated as mentioned below under"tomography. However, literature reports that in bronchography the cysts fail to fill with the contrastmedium.

\section{Tomography, "body-section- radiography" :}

This is of utmost importance in all groups, because the initial radiological diagnosis of a paragonimiasis often depends on the employment of tomngraphy. However, in paragonimiasis tomography of the whole chest is necessary, some times in different positions.

Spot-tomography as used in tuberculosis 
is only a secondary test. Especially, hidden changes only can be disclosed in tomography of the whole lung and ofte$n$ multiple changes which have not been suggested in the routine chest $\mathrm{X}$-ray are to be found. This is of importance for the decision whether a lobectomy or a bronchography should be emplnyed. We would say that usually both lobectomy and bronchography are contraindicated in paragonimiasis, because of the preponderance of multiple changes already shown in the tomogram.

\section{Differential diagnosis :}

All the above mentioned groups should give a glimpse into the variety of paragonimus-infection and its complications in the lungs. There are some, who conclude that those complications are only secondary diseases. Opposing such conclusions we present the fact, that those pneumothoraces as well as the pleurisies, empyemas, bronchopneumonic and central forms of the disease definitely demonstrate ova in the sputum and/or the fluids, while tuberculosis or other reasonable causes havebeen excluded by sputum, stool, fluid, cultures and skin-test investigations.

Contradicting the beliefs of many authors, we can state that the diagnosis of paragonimiasis and the differentiation against tuberculosis can be made with a considerable certainty already in the $\mathrm{X}$-rays as long as one has had experience.

Besides tuberculosis, the "malnutrition" is of special intererest in children. The $\mathrm{X}$-rays of the central form of paragonimiasis resembling a central pulmonary congestion can be very similar to those of a "malnutrition". The cooperation with the pediatrician of the Swedish HospitalDr. Vainola-enabled us to work out this most important differential diagnosis. The seriously ill children having edemas of the whole body including the lungs with a tendency to a beriberi stricken heart, clinically show clearcut condition.

They get quite better soon after corresponding therapy while paragonimiasis would not. The edema of the lung in the onset of the "malnutrition" on the other hand causes the findings in chest $\mathrm{X}$-rays with pleural shadows as well as central shadows.

Here we would like to mention that paragonimiasis patients very often have slight edemas especially of the faca which they often report spontaneously.

Next in differential diagnosis is the group of pneumomycosis. The initial onset of histoplasmosis, for example, appears with irregularly mottled shadows which become slowly spherical or ovoid nodular densities sometimes even combined with small cavities and pleurisy. Therefore the differentiation against paragonimiasis might be difficult. We reported at the Annual Medical Meeting in Seoul, May 1956, presenting very typical $X$-rays with the pathognomonic disseminated pulmonary calcifications, that histoplasmosis is most probably present in Korea. Some research in Sweden showed the evident miliary calcifications in the lungs but with negative skin test for histoplasmosis. Our cases here also were negative for histoplasmosis in the skin test. Theoretically this interesting correlation is not the place to discuss here. But we have to realize that $X$-rays in many diseases cnable us to the definite diagnosis with 
certainty-as also seen below in"toxoplasm. osis" under differential diagnosis of cerebrai lesions-, The same is true for histoplasmosis in which the fact has been proved by White, that cases of disseminated pulmonary diseases which go on to disseminated pulmonary caicifications can usually be attributed to histoplasmosis. Since we know that on the other hand in the majority of histcplasmosis cases the demonstration of the etiologic agent, (histoplasma capsulatum), has not been achieved (French, White etc, ) and that complement fixation test becomes negative at the time of or soon after calcification of the lesion(Campbell. Binkley), the X-ray diagnosis is of the same importance in histoplasmosis as shown in paragonimiasis. Interestingly histoplasmosis is also preponderant in males.

Of epidemiological interest is the report of Eung Soo Han, Seoul 1956, who said a suryey in 1953 did not reveal positive histoplasmin skin tests in Koreans. Should possibly exist another species in Korea?

In regard to calcifications in the lungs it is of particular interest that paragonimiasis shows no tendency to calcify even after a long course. Only one report in literature statas the suggestion of calcifications after therapy. But this statement of a few cases has not yet been approved by others.

In revers the paragonimiasis of the cerebrum develops often calcifications,

\section{Heterotopic paragonimiasis: cerebral clasification:}

Table I showed the completely surprisi$\mathrm{ng}$ fact that more than $25 \%$ of our cases $\mathrm{h}-$ ad cerebral complications. While literature describes crebral paragonimiasis as a rare heterotopic manifestation with only some 175 cases reported from 1887 until 1956, we found during our medical survey in an endemic area-mentioned above-13 cases with cerebral complications. This has been so stimulating to us that we started to investigate all our paragonimus patients for neurological symptoms.

The clinical symptoms have been various, depending upon the site and size of the crrebral lesions. Almost all patients complained about headaches, some times very severe ones, and often localized to a circumscript region. Very common have been epileptic səizures, cramp attacks. vomiting, dizziness, and unconsciousness. Single epileptic fits to repeated daily fits could be observed, The patients demons trated in a high number contralateral hemiplegias, some with only slight weakness but others with a fullblown spastic paralysis. Also cases with a so called hemiplegia alternans have been observed. Symptoms as such aphasia. speech-disturbances, visual disturbances, homonymous hemianopsia, diadochokinesis, astereognosis, ataxia and also mental disorder could be seen, Meningitic symptoms have been important in several cases.

There has been no constant pattern in the cinical course and development. But generally the disease had a slowly progressive character, causing death often within the first years and of ten unexpectedly without alarming symptoms. Literature reports that carebral involvement causes death usually within about two years. But we had cases with a history of more than 10 years.

Repated remissions and exacerbations are remarkable in the clinical course and 
are of certain pathognomonic importance for the cerebral paragonimiasis. All the various symptoms simulating meningitis, tuomrs, brain-abscesses etc. often lead to misinterpretation but indeed are commonly caused by paragonimiasis, especially in Korea.

The routine skull $\mathrm{X}$-ray demonstrated only in $50 \%$ of our cases evident changes. Intracranial calcifications in 29\% and enlarged sella in $21 \%$ have been most helpfull findings (table 1). The calcifications mostly are localized near the brain surface and often to be found in the parieto -occipital region. We could also find calcifcations near the frontal skull base or hidden in the middle skull fossa.

Tomography is just as helpful in both the localisation in depth and the demonstration of hidden calcifications. We have had patients in whom only the tomogram could outline the calcified lesion.

As to when cerebral lesions become calcified we had one interesting case.

During the first onset of clinical symptoms-several "meningitic attacks" -two years ago the routine skull $x$-rays did not show any pathologic changes. Now, two yaars later, we could demonstrate a group of definite calcifications in the left paramedian cccipital region. The 10 years old girl had a slight weakness of the right arm, a homonymous hemianopsia and now the first heavy epileptic seizure of Jacksonian type.

In contrast to this case we had other patients with longstanding clinical symp. toms for many years but no calcifications at all.

Another help in the routine skull X-ray is an enlarged sella which we found in 21 $\%$ of our cerebral cases. Calcifications and enlarged sella together we had only in one case. Because of the progressive chronic intracranial process it is so understand that the sella can become enlarged due to intracranial abnormal pressure. In the same regard we understand the appearance of increased "digital impressions" in some of our patients.

The pneumoencephalography shows striking changes even in the absence of calcifications or enlarged sella. General dilat ation of the ventricular system has been found as well as the pictures of spacecccupying lesions with heavy shift of the ventricular system. Also aspects like shrinking inflammatory processes with traction of the ventricular system have been present. And in both circumstances the ventricular system can also become correspondingly narrowed by the lesion.

The cerebral arteriography has been made only preoperatively in a few cases and showed changes in the pattern of the cerebral arteries.

Due to inadequate facilities we have not been able to perform both the pneumoencephalography and the cerebral arteriography in all our cases.

Only a few have been oparated by the neurosurgeon Dr. Kim Sun Keun. As to the question of an active neurosurgical program experience so far has failed to prove if operation should be done in all cerebral cases and in which stage. We feel with our limited means that some cases show a contraindication for a neurosurgical procedure. This is true probably for the earliest stage, the meningitic forms, the multiple lesions and may be also for some very advanced cases. The chronic course gives time enough for a careful observation and 
investigation, before the deciison on an oparation.

On the other hand, the operation undoubtedly is of important help in many cases. Literature in single cascreports describes excellent results after operation. For different reasons a successful operation is of importance because most of our patients $(75 \%)$ are less than 30 years old and $43 \%$ are less than 15 years old, Sex-distribution was $79 \%$ males.

Our assumption of an unexpectedly high number of cerebral paragoniniasis in Korea is furthei aided by the increasing number of cerebral cases observed within the last two years by the neurosurgeon Lee Choo Keoul who told us about 40 cases operated by him and neurosurgeon Kim Sun Keun who apparently observed more than 20 cases, Ourselves we observed 65 cases within the last three years. Compared with the total of some 175 cases throughout the world since 1387 there have been in Korea within only the last three years at least 125 new cases. And this number would easily increase if some hospital facilities and hospital economies could be improved.

Additionally the redent papers of Mitsuno and Zimmerman reporting about 11 carebral cases from Japan prove that carebral paragonimiasis is in other countries stressingly widcspread, too.

\section{Differential diagnosis :}

The variety of both clinical symptoms and the radiological findings in carebral paragonimiasis simulates many cerebral conditions as montioned above, This proliminary report shall explain about conditions which we also believe necassary to be taken into consideration.
In single case-reports in literature as well as in our observation it is striking that all symptoms of a meningitic condi tion were observed.

Therefore many times the misinterpretaticn of tuberculous meningitis has been made but this was understandable. The differentiation needs extremely careful and repeated investigations in all diagnostic fields. But it also needs the knowledge of paragonimiasis itself and especiait $y$ ot the pulmonary manifestitaon, sinc analogically the same is true tor tuberculosis and for paragonimiasis that a manifestation in the lungs proves indirectly the crigin of the carebral lesion.

Literature on spinal fluid describes ery ditferent findings which are apparently inconsistant in comparing the single case -repots, For example the presence of eosinophil cells is not at all oblgatory in carebral cises a fact which we have also found in our cases.

Inconsistancy is the same in the sugar level of the spinal fluid. It is not unuswal that in the sam? patient the sugar 1 evel shows repaated fluctuation from norm al to decreased and even 7ero percentage.

Moreover the colloidal gold curve shows aiso such fluctiations,

We have observed that the curve can change within 4 weeks from a normal to a pathologic curve. But it seems that the curve of the paretic typa was predominating, as observed-not yet publishedby the Mary Knoll Sisters Clinic, cooperating with us in pusan, but already published by Mitsuno, Japan.

Additionally the color of the spinal flu id can change from a clear normal fluid to a cloudy yallowish color and even pus like fluid, with more than 2000 cells. The 
presence of ova is unusual.

The complement-fixation test of the spinal fluid we shall discuss below.

Toxoplasmosis is another disease of differential diagnostic importance.

Its calcifications can resemble that of paragonimiasis. Through literature it has become evident that cerebral calcifica tions of a symmetrical as well as of an irregular multiple type are often attributed to the late stage of a cerebral infection with the protozoan "toxoplasma".

Comparable to the above mentioned relations in histoplasmosis we have to realize that $x$-ray investigationsis the decisive factor in the diagnosis, because the color titer (Sabin-Feidman) as well as the complement fixation tast and the intracutaneous test $c$ an be negative.

We saw Korean patients with the typical symmetrical calcifications in the choreoideal plexus (glomi choreoidales) and we feel sure that toxoplasmosis is to be found in Korea.

\section{Other heterotopic localisations}

In our cases only a few patients with subcutaneous nodules, involvement of the scrotum and of the abdominal cavity have been observed. These patients did not show any important differences to those in literature.

But we like to point out a fact which might give an explanation as to why literature reports that many times the parasites have not been found in the removed nodules. In all our patients the subcutaneous nodules were very difficult to outline against the surrounding tissue during surgery. They had not been always connected by burrows, and it was necessary to remove relatively much of the surrounding tissue. The search for the parasite in all specimens therefore was very difficult and it took 30 minutes and more to find the parasite, On the other hand we have only found parasites smaller than the size of a real adult fluke averaging about $1,5 \mathrm{~mm}$ to $3 \mathrm{~mm}$, In color the parasites appeared like soft tissue. Therefore the search always has been so difficult that we feel that it is pos sible to never discover the fluke. When local anesthesia was employed this diffi culty increased,

\section{Differential diagnosis:}

Regarding the subcutaneous nodules we observed similar changes caused by parasites of the sparganum-group. This entirely different parasite is of further interest because subcutanenus nodules, as well as changes in the lung and pr obably also intracerebral changes simulate almost exactlythe condition of a paragenimiasis. We had a patient where some subcutaneous nodules. a pleurisy and a questionable intracranial calcification were observed. Repeated sputum examinations as well as a skin test were negative for paragonimiasis and finally the operation proved the "sparganosis" with the parasite in the nodule.

The paragonimus skintest will be expl ained below, The "sparganosis" shall be published in another paperafter further results,

\section{Distribution of heterotopic paragonimiasis}

In this rogard the following reports in literature are most interesting: from the Philippines more cases with abdomincl involvement; from Formosa 10\% subcuta- 
neous nòdules; from Japan some cerebral cases and from Korea a few abdominal and cerebral cases have been reported bef ore 1955, Such different findings could be caused by different types of parasites but most probably are caused by a different approach by the investigators.

So it was obvious that in our hospitals the more our experience increased the more cerebral cases, subcutaneous, nodul es pleurisies etc. were discovered.

Of special.interest is a recent report by Chae, Yun, and Soo-Severance Medical School, Seoul-about two cases of paragonimiasis in the spinal cord, which might be the result of an increasing interest in this important disease.

The extremely rare localisation in the urinary tract-reported by Iwasaki and by Weinstein-gives another hint of a heterotopy,

As a rule paragonimiasis is not a "generalising disease" as maintained by some authors, but a condition prefering the lungs and with the possibility of heterotopic multiple localisations,

A difficult but most important question is whether a unilocular heterotopic manifestion is possible, especially in the cerebrum, Literature describes single autopsies with only cerebral involvement, But at least some of these reports seem doubtful to us since they describe for example "pleural reactions, but no parenchymal changes or parasites" in both lungs, According to our above mentioned systemic investigations bilateral pleural reactions are highly suspicious for paragonimiasis,

We do not have a definite unilccalized case and neither do we have a so called "X-ray negative" case of the lungs in our cerebral cases,

However, unilocalized heterotopic paragonimiasis is theoretically to be considered and might be possible to discover with the help of a skin test and/or a complement fixaiton test for paragonimiasis,

\section{Paragonimus skin test:}

The diagnosis of paragonimiasis:depends on circumstances and hospital facilities which are not always available in Korea and some other countries.

Therefore it was important that a skin test be developed and employed by some authors such as Ando, Ritchie, M. Yokogawa or Chung. The material for such a skin test unfortunately is not yet generally available but has been supplied to us with the kindness of Dr, Rietchie, (406 th Gen. Med. Lab. U. S. Army, Tokyo) and Prof. M. Yokogawa. Japan. The e xtensive research of Yokogawa excludes with some certainty a cross reaction to other trematodes such as clonorchis sinensis with this skin test material. Fortu nately we can agree with Ritchie and Yo kogawa and can report that all our case $\mathrm{s}$ which had ova in the sputum also had a definite positive skin reaction with the exception of one case; but this one had a positive complement fixation test in the blood-serum. We had slso a few pat ients who had a Positive skin test only with a dilution of $1: 1000$ while $1: 10000$ showed a negative reaction in the same Patient. We hope to be able to study these cerrelations in a further research since we could not employ these methods in all our patients.

Complement fixation test (CFT): 
With the kind assistance of Prof. $M$. Yokogawa we have started to employ this method on some of our patients. We have controlled it especially in cerebral cases. In this regard Chung reports that the CFT in the spinal fluid is positive in cer ebral paragonimiasis. We have to report that in our cerebral patients the findings conflict with those of chung.

The CFT of the spinal fluid has been mostly negative but the skintest in the same patient as well as the CFT in the blood-serum were positive.

We had one case in which the CFT of the' spinal fluid changed within 3 weeks from a positive result to a negative. The patient had very aeute clinical symptoms with a meningitic condition; all investigations for tuberculosis were negative, except the skintest on the 14 year old girl; all investigations for paragonimiasis were positive such as chest $\mathrm{X}$-rays, sputum, skintest, CFT in the blood-serum and CFT in thes spinal fluid during the first stage of the disease. Three weeks later the spinal fluid showed a negative CFT.

\section{Therapy :}

The problem of treatment certainly is the most vital issue. But to date no one has approached it systematically with a certain effectivity, As mentioned above paragonimiasis until now was not believed to be a too serious condition. This misinterpretation might explain the lack of extensive studies in therapy. The previously reported therapy with emeti fective has been employed by others and also in our hospitals but apparently failed to cure the disease. We ourselves have tried in some cases Chungs therapy of high dosage of chlorequine up to $80 \mathrm{~g}$ within 3 months: but we have combined the drug with hydantoine due to an obse rvation in cerebral patients that both the general condition and the result in the sputum have improved only by the employment of hydantoine. Howaver, the experience with this therapy is not yet extensive enough for any conclusions. But we anticipate that chlorequine will not be effective.

\section{Final conclusion :}

311 patients with pulmonary paragonimiasis and a great number of them with an additional extrapulmonary paragonimiasis have been thoroughly studied. The results have been presented in some previous lectures, (below*), together with the demonstrations of $\mathrm{X}$-rays and $\mathrm{a}$ movie of parasitological studies as well as animal experiments and demonstration of patients in this movie. On the whole the main-concept is that, contradicting the previously held beliefs, paragonimiasis produces many times very severe conditions and that an unexpectedly great number of patients die from the disease. most probably in early childhood, with the true diagnosis unrecognized.

We can therefore state that this parasitic infection represents a considerable medical, sociological and economic prob. lem at least in Korea.

$\mathrm{n}$, sulfonamids etc. believed. to be ef-

*) 1. Tokyo , Japanese Annual Radiological Congress, 
3. Pusan, International Med. Society, Swedish Hosp, June 1956

4. Mexico , Eighth Internatinal Radiolog. Congress, July 1956

5, Washington, Walter Reed Army Institute of Research, August 1956

6. Boston , Harvard Med.School, Peter Bent Brigham Hosp, , August 1956

7, Aarhus, Scandinavian Congress of Neurosurgery. September 1956

8. Berlin, University Hospital, Berlin-West, September 1956

9. Geneva, World Health Organisation, September 1956

10, Manila, Philippine Tuberc, Soc, Quezon Institute, September 1956

11. Pusan, Annual Meeting of Med. Missionaries-Korea, October 1956

12. Seoul , 38 th parallel Medical Society of Korea, January 1956

13. Seoul , Severance Medical College. January 1957

14. Seoul , Universicy Hop. of Seoul Nat. University, February 1957

15. Taegu, Symposium of Leprosy and paragonimiasis,

Taegu Med. College, Kyong Buk Nat. Univers.,

February 1957

**) Further reports in detail will be published with $\mathrm{X}$-rays etc.

Pusan, March 26. 1957. 